\title{
Process Monitoring and Evaluation
}

Dr. Tobias Wille, Falk Heinecke, Robert Hein (German Aerospace Center - DLR)

Self-Sensing Composites - Public Event Ghent, 7 February 2017
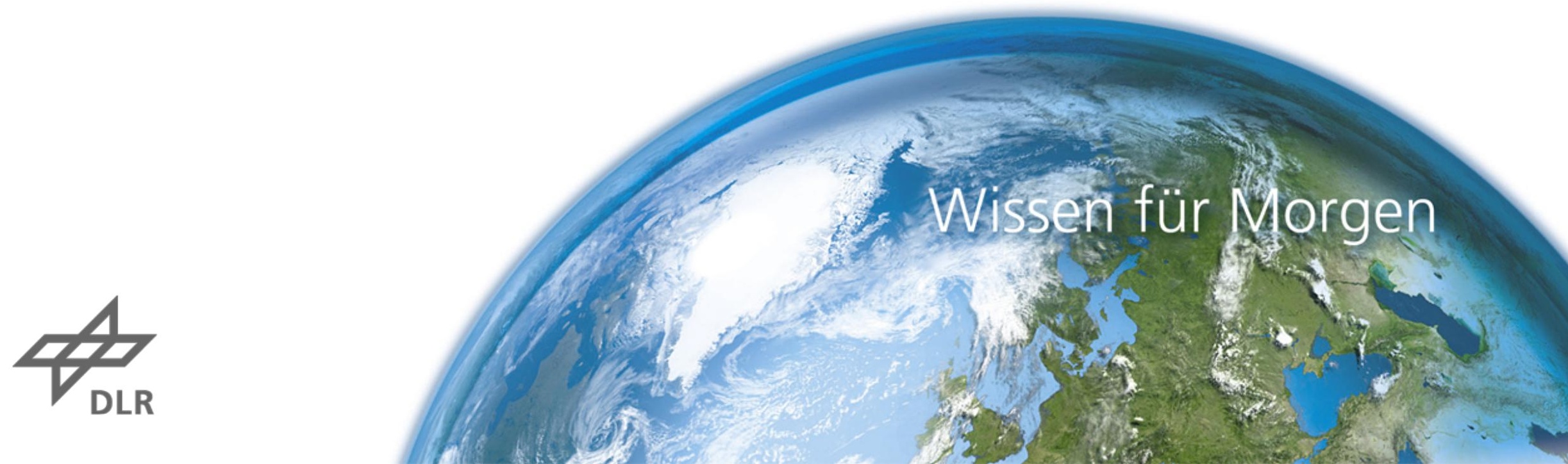


\section{Outline}

- Motivation

- Fibre deposition monitoring

- Example for in-situ structural evaluation of fibre deviations during AFP

- Resin infiltration and cure monitoring

- Example for in-situ structural evaluation of process induced distortions

- Conclusion 


\section{Motivation}

Potentials of Composites

- High weight specific mechanical properties

- Integral Design

- Anisotropic Tailoring
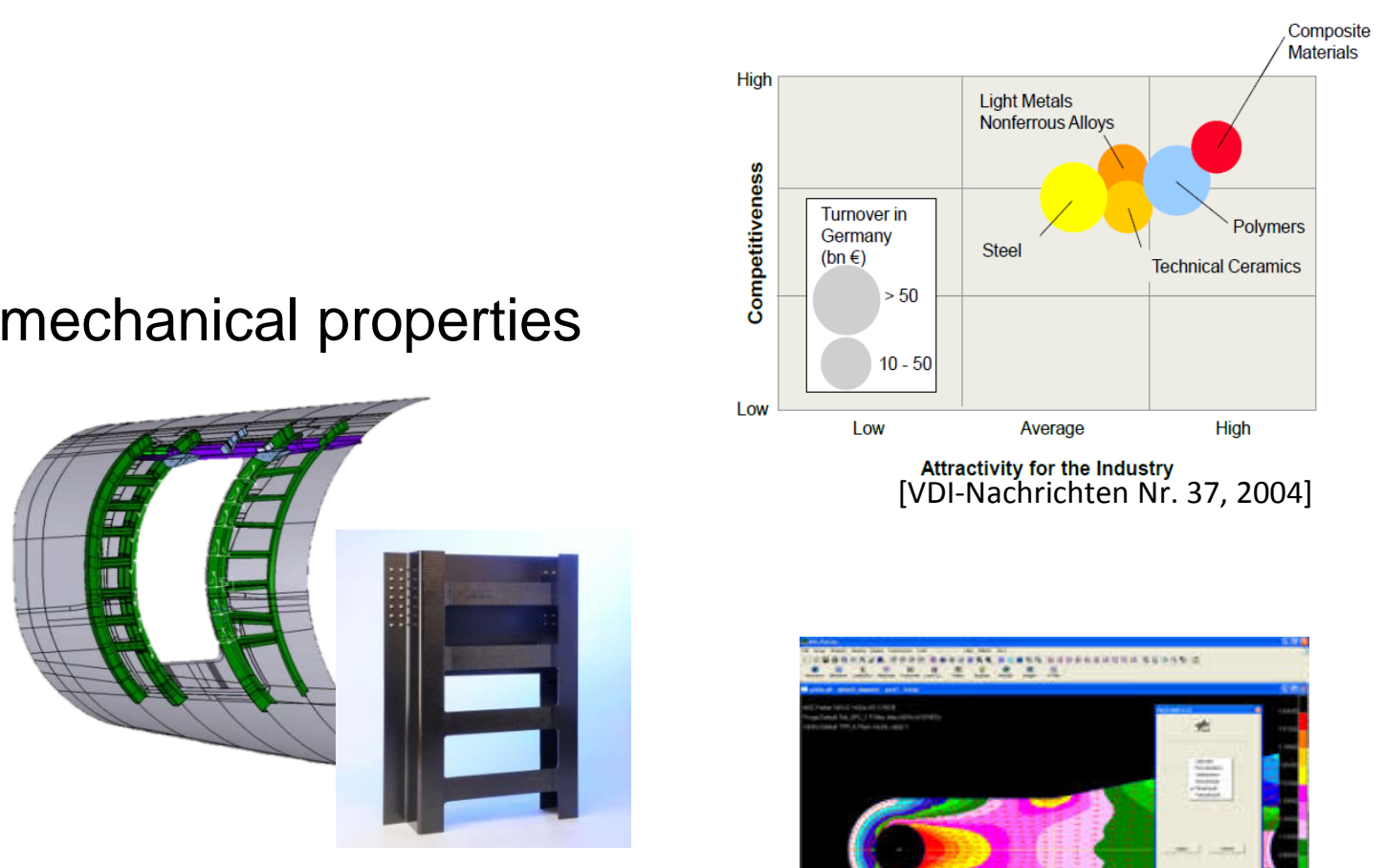

- Function Integration
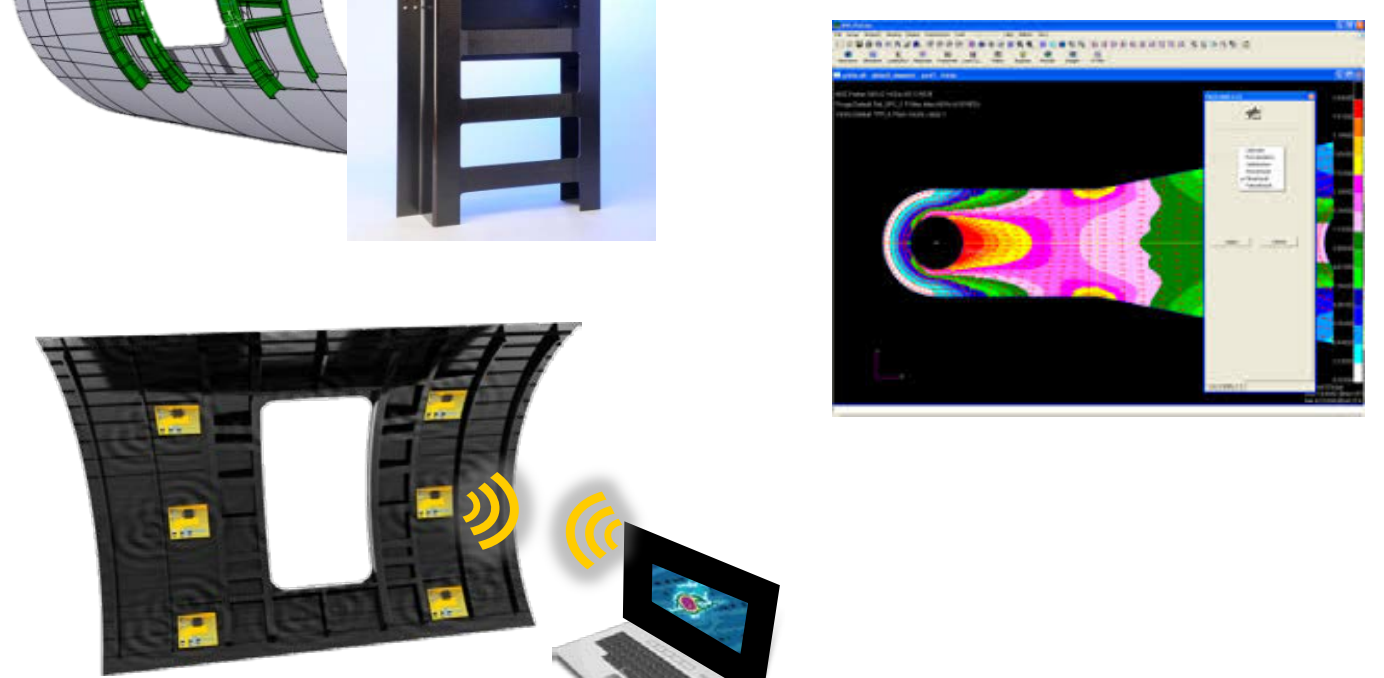

- $\quad \ldots$ 


\section{Motivation}

Great variety of composite materials and manufacturing technologies

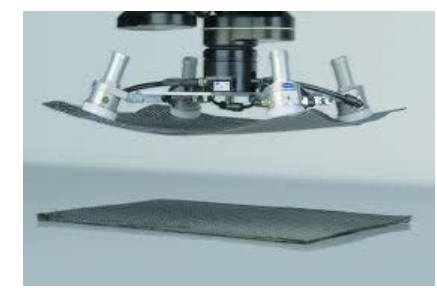

Pick\&place [Schmalz]

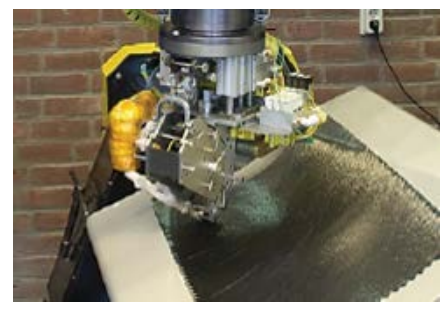

AFP [NLR]

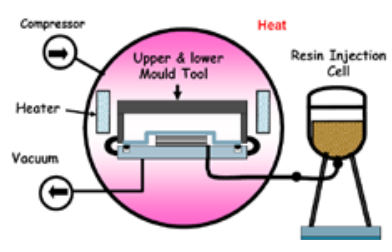

$\mathrm{RTI}[\mathrm{BAB}]$

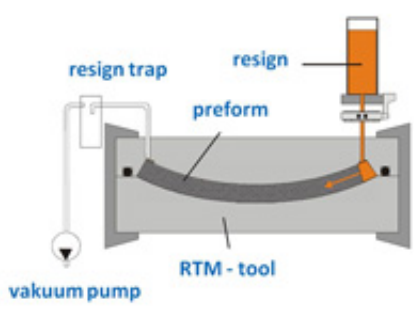

RTM [DLR]

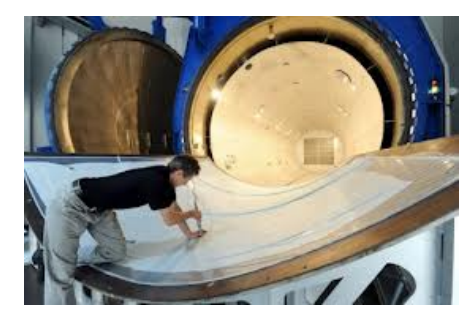

Autoklav [DLR]

Property development during manufacturing, depending on material systems, process technology, process parameters
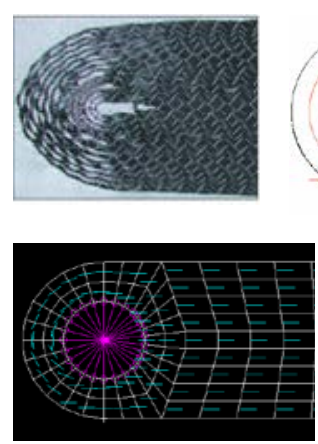

Fibre orientation resulting from dry fiber placement [DLR]

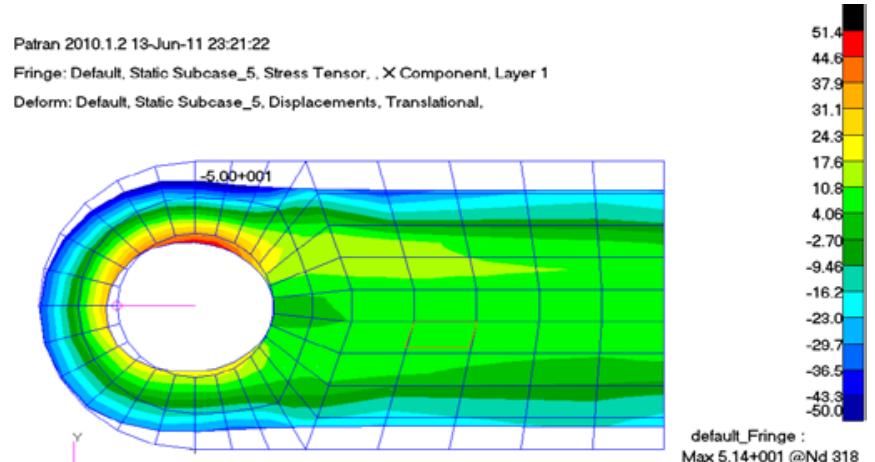

Residual stress in fiber direction [DLR] 


\section{Motivation}

Inevitable uncertainties

- Material properties and tolerances

- Process parameters and tolerances

- Inevitable deviations or faults

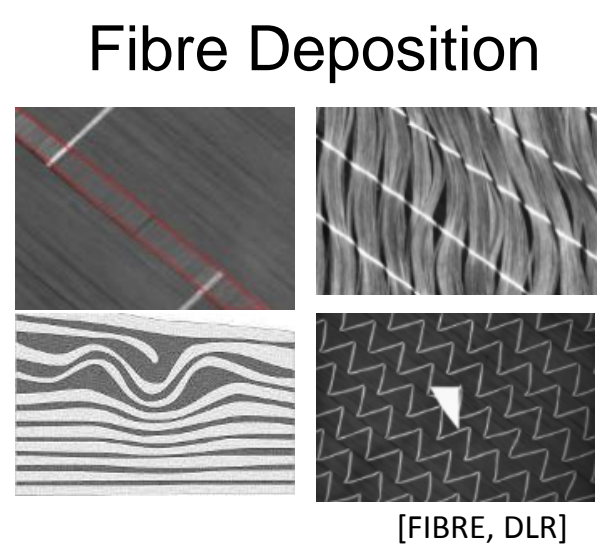

- Fibre orientation

- Waviness

- Gas, Overlaps

- Foreign objects

- ...

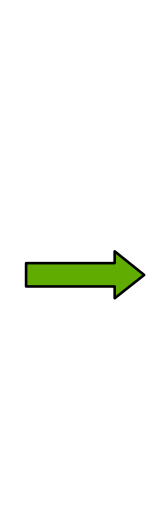

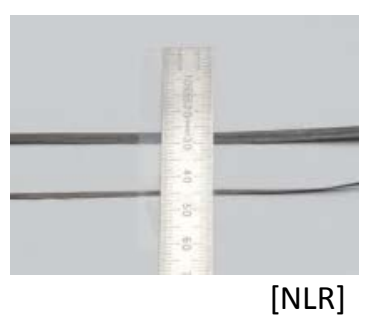

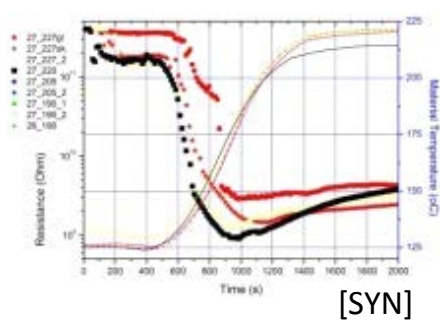

Resin Infiltration

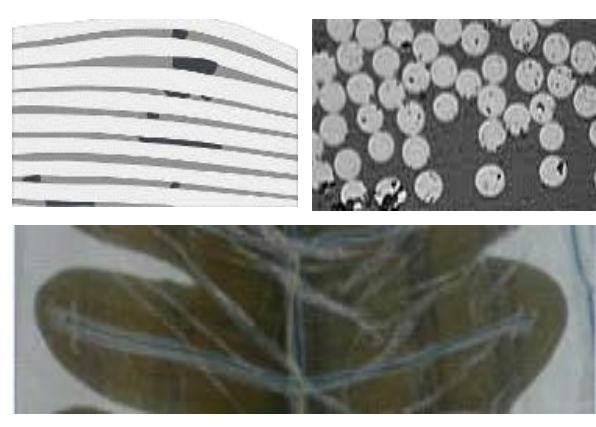

- Fibre volume variation

- Pores

- Resin rich areas

- Air entrapments

- ...

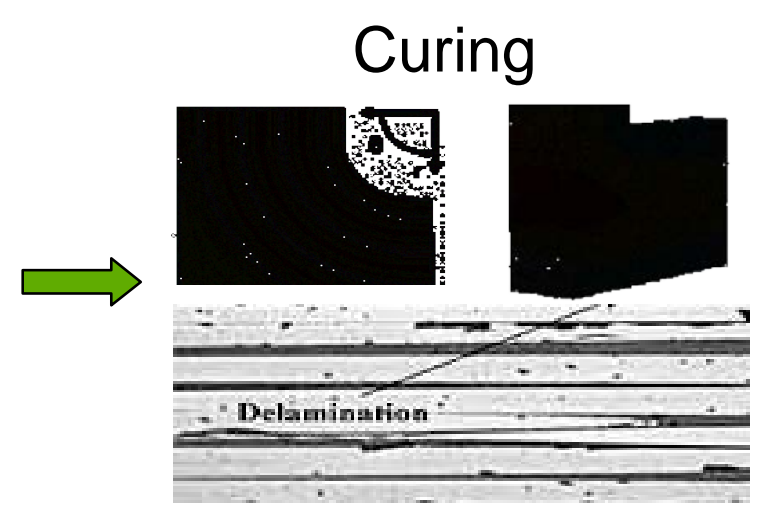

- Overheating

- Degree of cure variation

- Chemical shrinkage, distortion

- Residual stresses, delamination

- ... 


\section{Motivation}

Effects of process deviations on subsequent assembly and operation

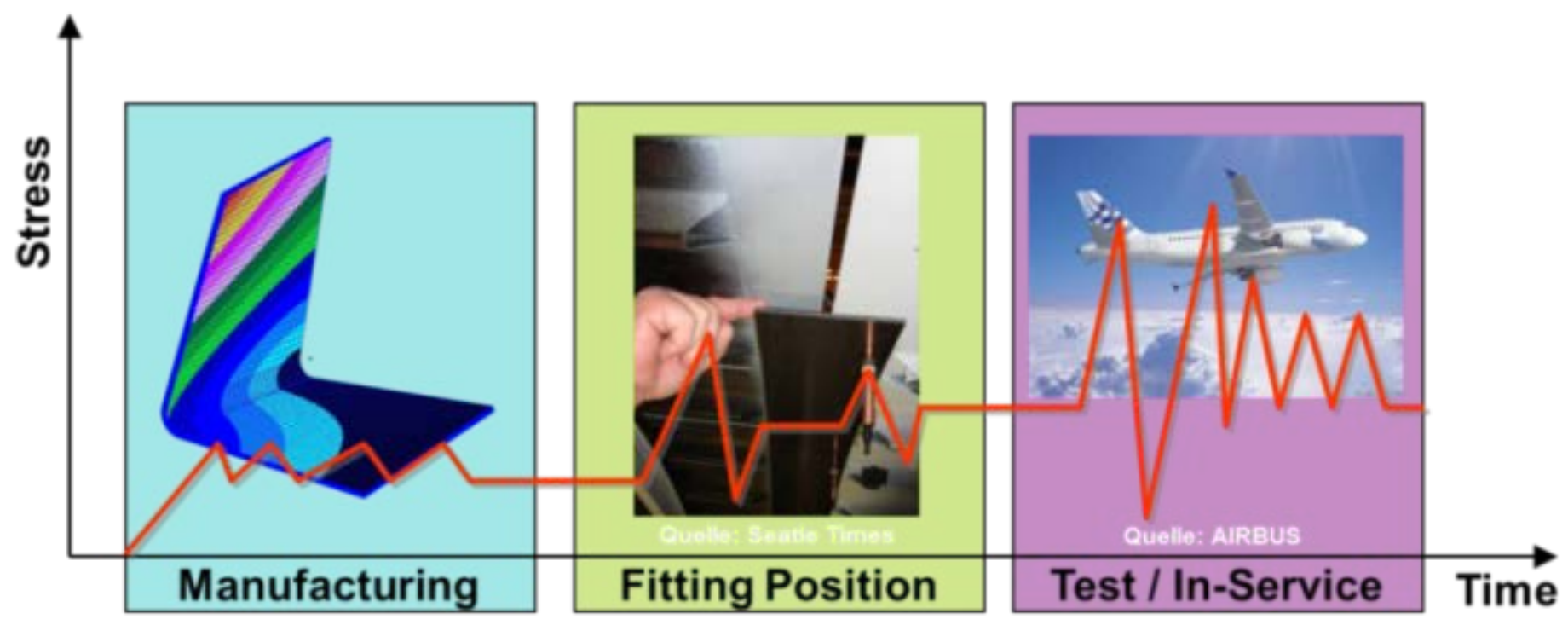

[Spröwitz et al., 2008] 


\section{Motivation}

Current state-of-the-art tolerance management

- Process design

- Mainly knowledge based

- Selected process simulations

- Conservative composite engineering requirements/ allowable

- High trade-off between accuracy and efficiency

- Process control

- Wrt. predefined global (conservative) process tolerances

- Few sensors within autoclave/ oven, tooling, traveler coupons

- Not considering actual structural properties

- Resulting in

- Conservative processing

- High non-added value costs in case of non-conformities 


\section{Motivation}

New tolerance management for efficient production requires

- Robust and reliable sensors

- Low complexity, easy to use

- Not affecting manufacturing process or structural properties

- Efficient analysis methods

- Real-time capability

- Multidisciplinary coupling of process and structural analysis

Potentials

- Increased understanding of materials and processes

- Significant reduction of process time, energy, emissions and costs

- Material savings

- Enhanced NDI

- Less rework and concessions

- Higher exploitation of composites

- Less maintenance effort, increasing life-time

- ... 


\section{Fibre Deposition Monitoring}

Depending on

- Part complexity (double curved, inserts, etc.)

- Fibre material (dry or prepreg, tow, tape, fabric, etc.)

- Manfacturing technologies (manual layup, pick\&place, AFP, winding, etc.)

Particular features to be identified and evaluated

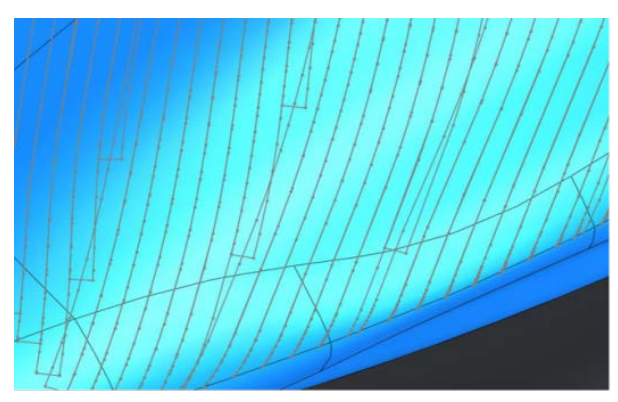

Geometrical induced features during AFP [Gerrits et al., NLR]

- Global and local fibre orientation

- Ply/ patch/tow boundaries

- Misplacement

- Thickness

- Gaps and overlaps

- Twisted tows

- Splicing

- In-plane/ out-of-plane waviness

- Foreign objects
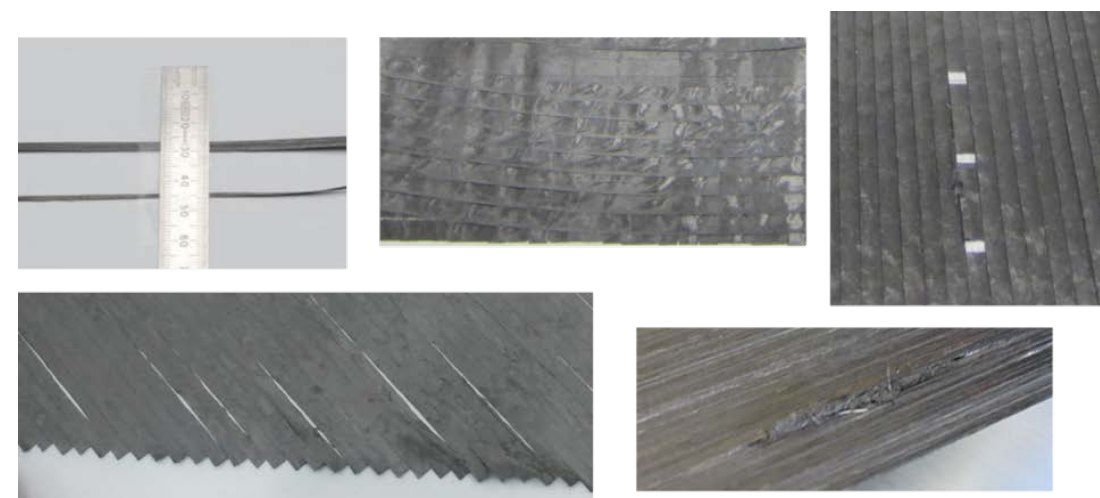

Typical defects during AFP [Gerrits et al., NLR] - ... 


\section{Fibre Deposition Monitoring}

Fibre monitoring by 2D image analysis

- Image quality depending on camera (lens, sensor), illumination, speed and fibre material

- Analysis of features depending on image quality and data analysis software
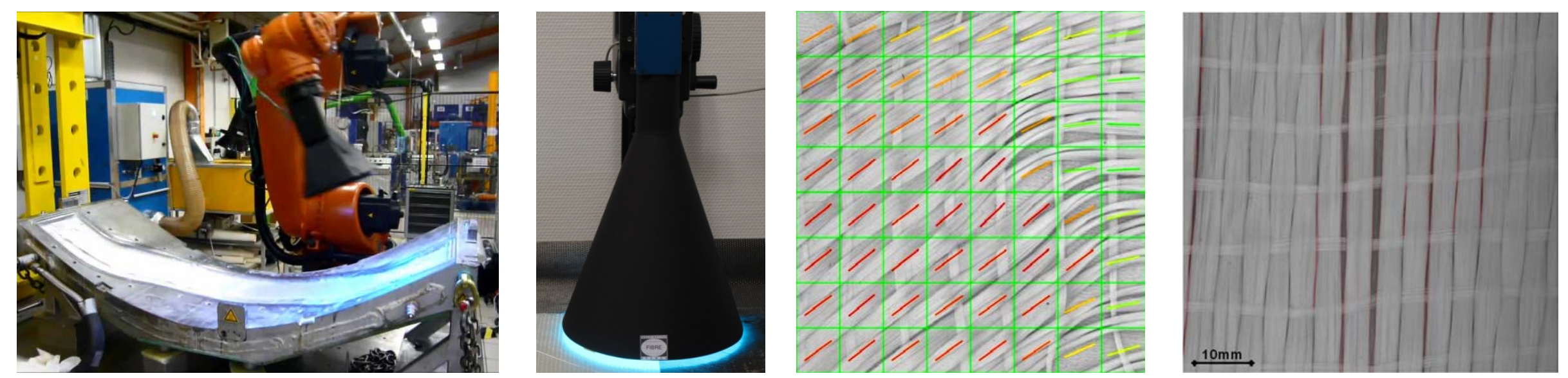

Suspension blade measured with diffuse light cone from Faserinstitut Bremen at Hutchinson [Miene et. al] 


\section{Fibre Deposition Monitoring}

Fibre monitoring by 3D laser triangulation

- Thickness profile measurement

- Data analysis (reference plane, strip stitching, smoothing, feature identification)

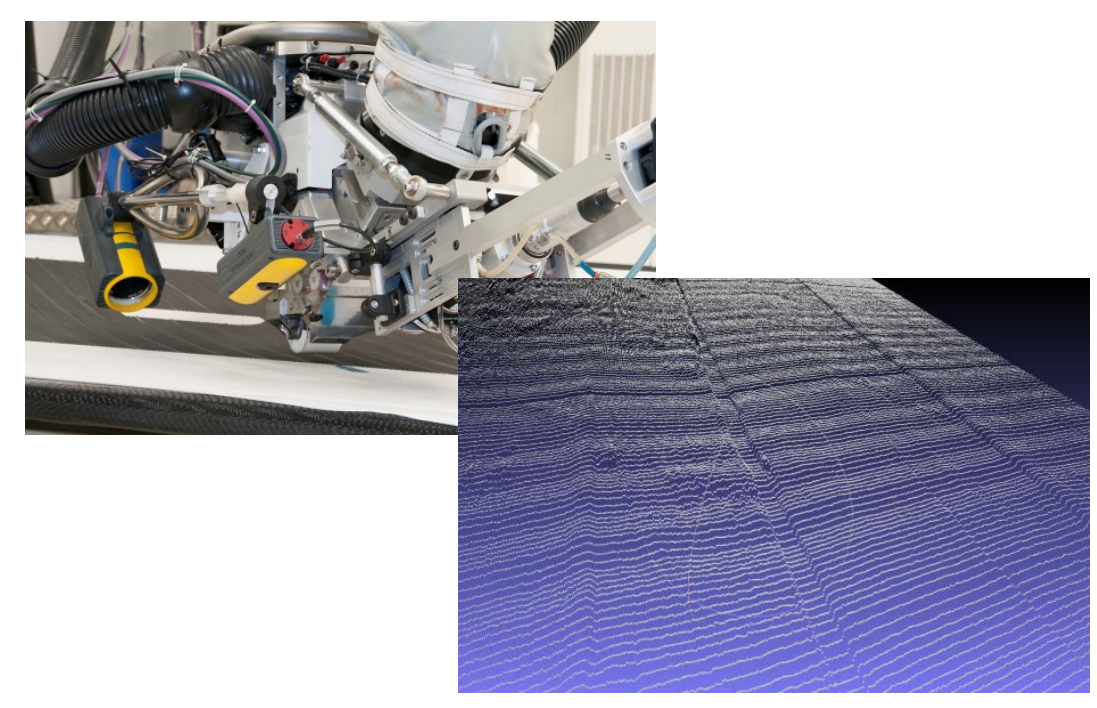

Wing Cover measured by Loop Technology during AFP at NLR [Redman et al.]

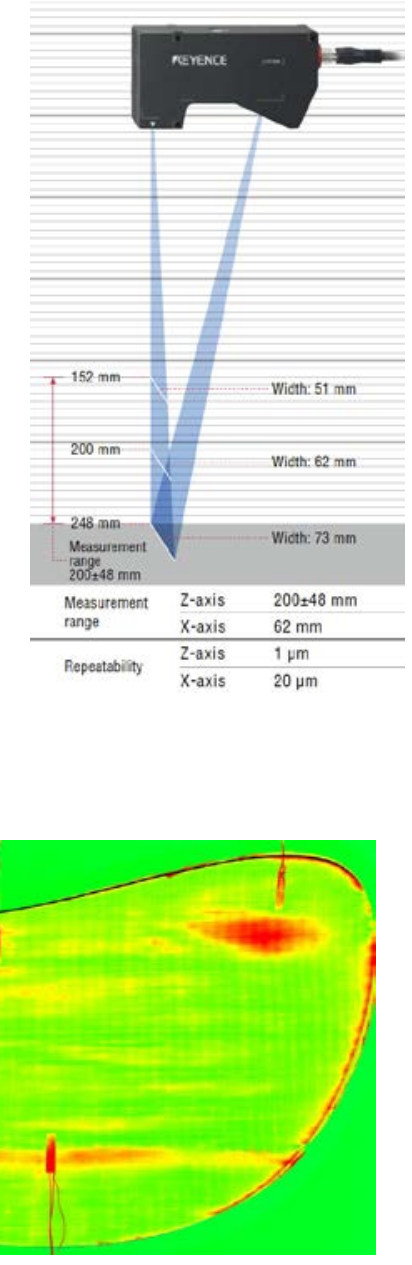

Ship Propeller measured by Faserinstitut Bremen [Miene et al.] 


\section{Fibre Deposition Monitoring}

During measurement

- Feature storage within manufacturing database and digital life data sheet

- Direct feedback to CAD/CAE for detailed structural analysis possible

Measures in case of deviations

- Remove foreign objects

- Ply correction in case of wrong orientation, misplacement, waviness, etc.

- Remove splices, fill in tows

- Add/ remove ply or core for thickness adjustment

- (Stop process)

Impact

- In-situ NDI (fibre architecture)

- Less conservatism required (engineering + manufacturing)

- Earliest correction to save waste of subsequent manufacturing steps

- Increased robustness for subsequent processes (e.g. reproducible runners and permeability during infiltration) 


\section{Fibre Deposition Monitoring}

Challenges

- Sensor robustness under environmental conditions (e.g. illumination, speed, vibrations)

- Sensor system integration into manufacturing environment (fibre deposition machine, work flow, software, database)

- Robust defect identification also for interacting defects

- Handling of material and process particularities, e.g. debulking

- Big data issues

- Real-time data analysis 


\section{Example:}

In-situ structural evaluation of fibre deposition of a composite wing (ECOMISE project)

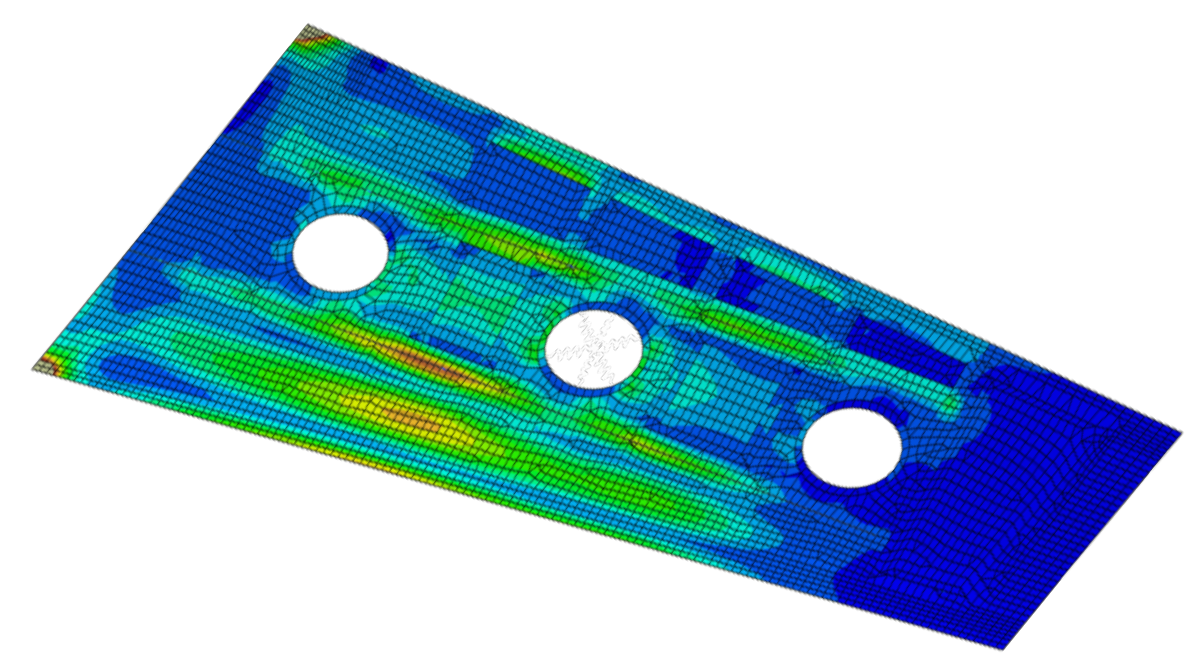




\section{In-Situ Structural Evaluation during AFP}

\section{Procedure}

1. Prior to manufacturing

- Investigation of effects-of-defects

- Database and surrogate modeling

2. During manufacturing

- Online process monitoring

- Feedback of online fibre measurment

- Structural model update and assessment

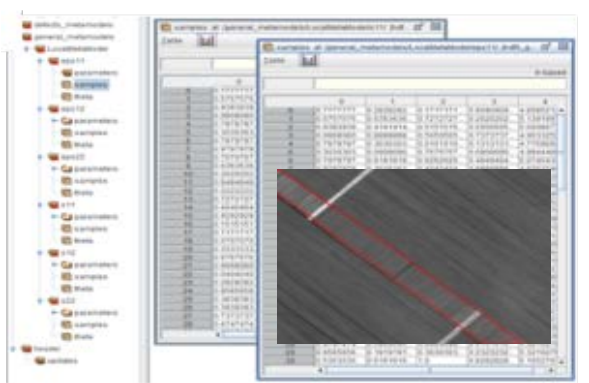

Surrogate Models Effective properties

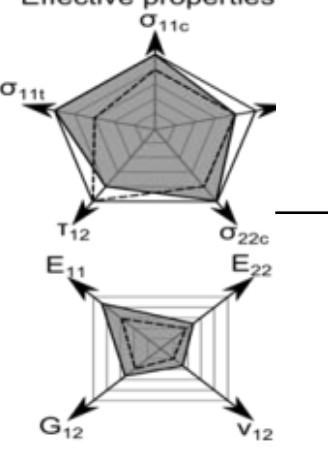

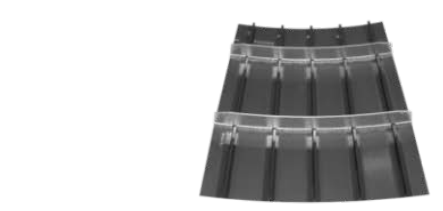

In-Situ

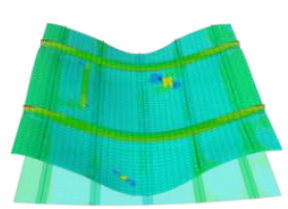
Feedback to Manufacturing

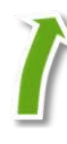

Strutura

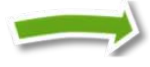

In-Situ Structural Evaluation via Manufacturing Database (Re-)Evaluation
Online

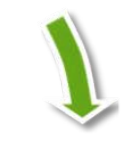

Effects-of-Defects Assessment

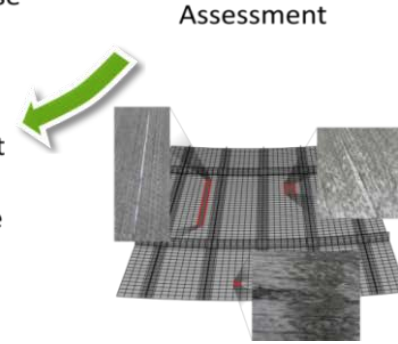

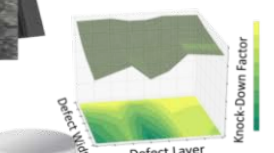

Effects-of-Defectect Daver

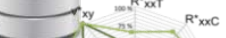<smiles>C1CC1</smiles>

[Heinecke et al., DLR] 


\section{In-Situ Structural Evaluation during AFP}

Industrial application (1. step, offline)

- Nominal design and analysis (without defects)

- Effect-of-defect analysis and surrogate models for knock-down factors on ply level
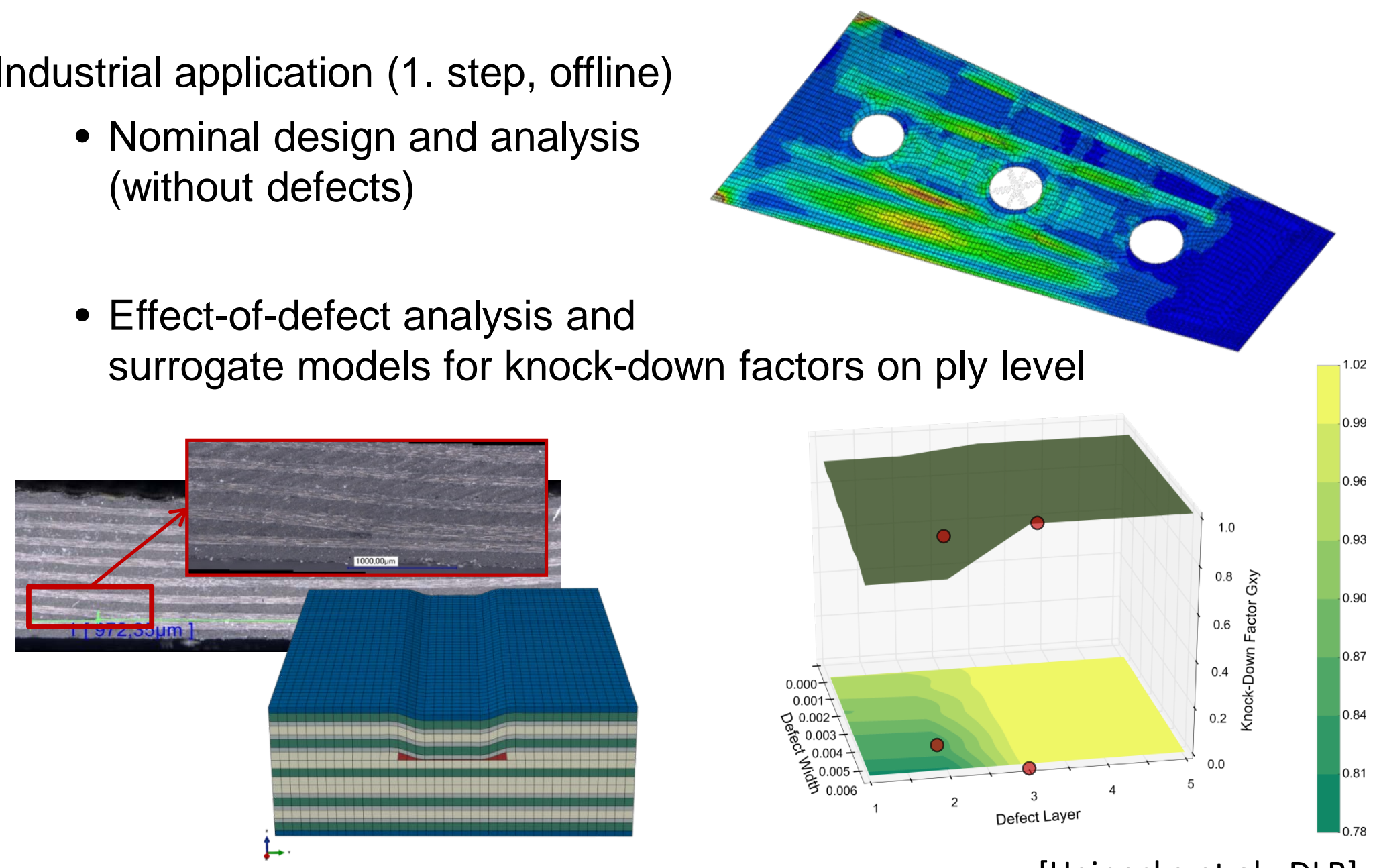

[Heinecke et al., DLR] 


\section{In-Situ Structural Evaluation during AFP}

Industrial application (2. step, online)

- Process monitoring

- Laser triangulation

- In-situ profile analysis and defect identification

- In-situ structural evaluation

Missing Tow

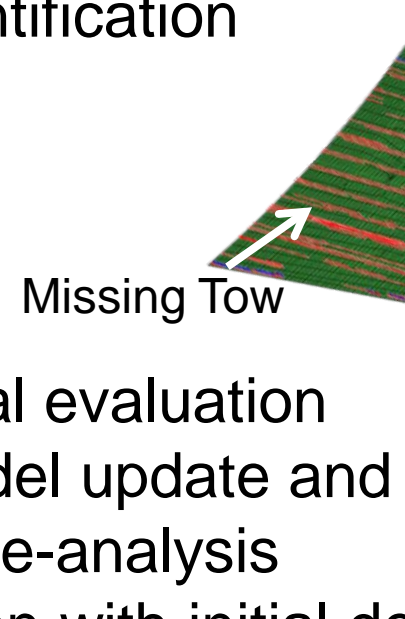

- In-situ model update and structural re-analysis

- Comparison with initial design and requirements

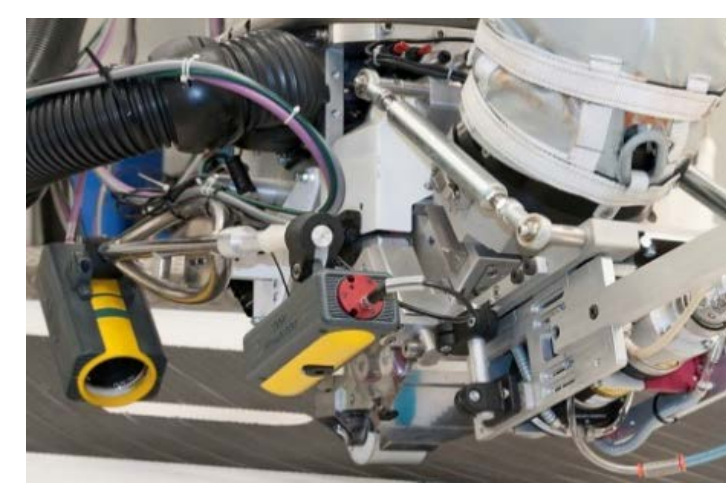

Measurement with Loop System at NLR

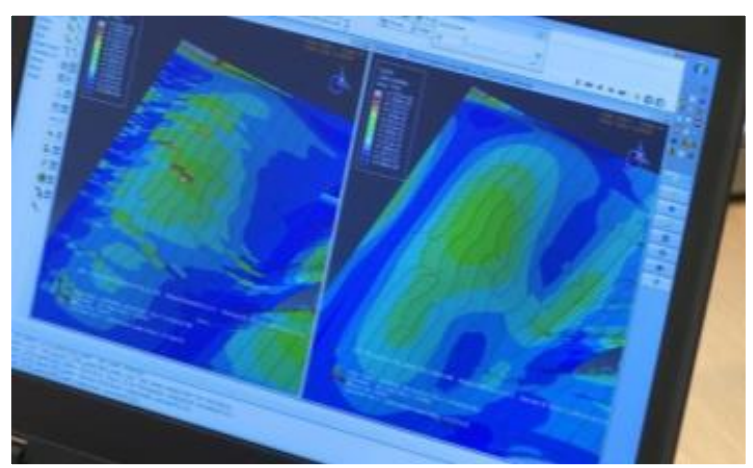

In-situ structural evaluation during AFP [Heinecke et al., DLR]

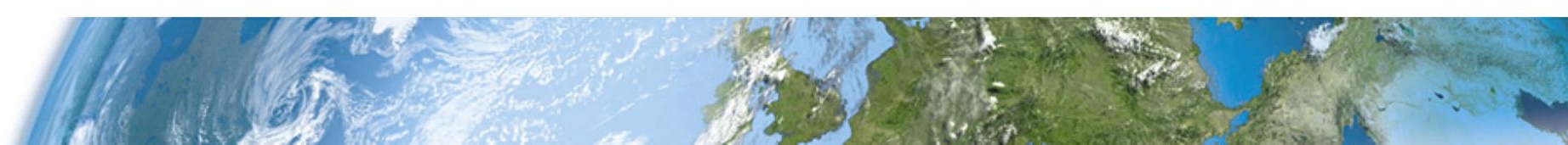




\section{Resin Infiltration and Curing Monitoring}

Typical infiltration and curing technologies for dry fibres and prepreg

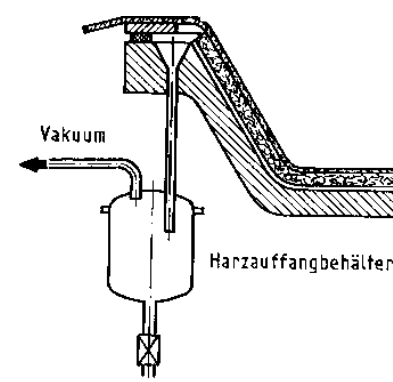

Vacuum bagging

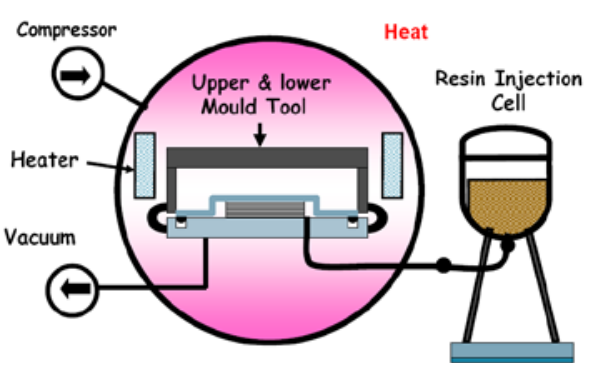

RTI

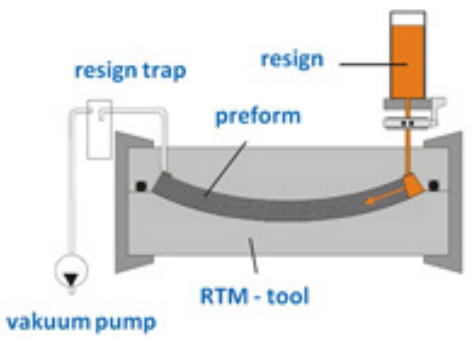

RTM

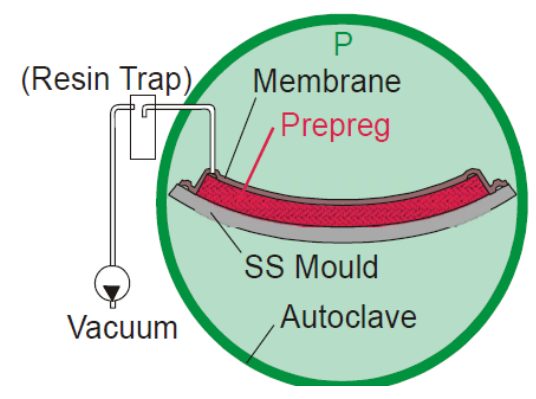

Autoclave

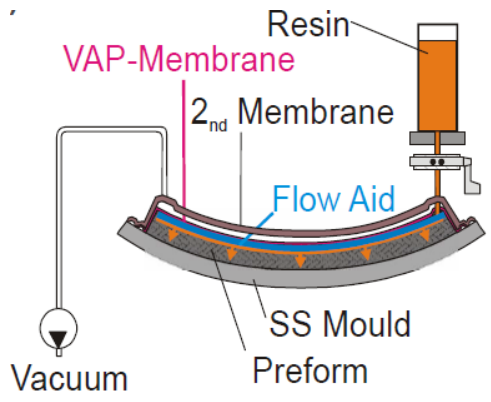

VAP

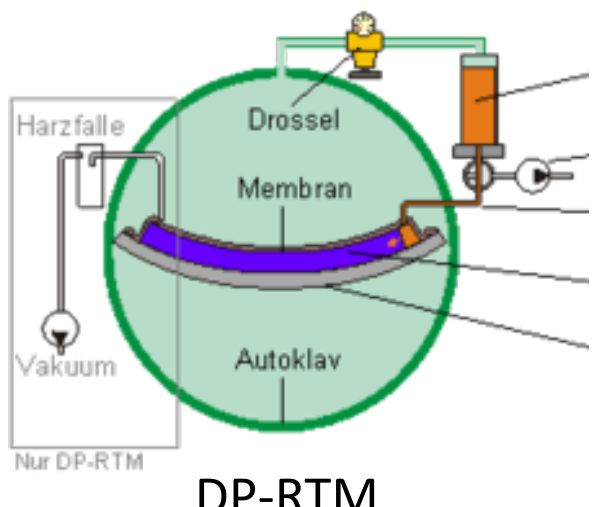




\section{Resin Infiltration and Curing Monitoring}

Monitoring systems required for

- Fast ramp-up through early and efficient identification of issues

- Sensor-based process optimization (e.g. during curing)

- Verification and validation

- Quality control

Parameters to be monitored

- Temperature

- Viscosity

- Pressure

- Amount of resin infiltrated

- Flow front, velocity and shape, air entrapments

- Degree of cure, point of gelation, glass transition

- Thickness/ fibre volume content and floating/ sliding

- Strain, stress

- Geometry 


\section{Resin Infiltration and Curing Monitoring}

Typical resin/ composite monitoring systems

- Thermocouples, thermography

- Pressure sensors

- Dielectric sensors

- Electrical resistance sensors

- Ultrasonic sensors

- Fibre optical sensors

- Digital image correlation
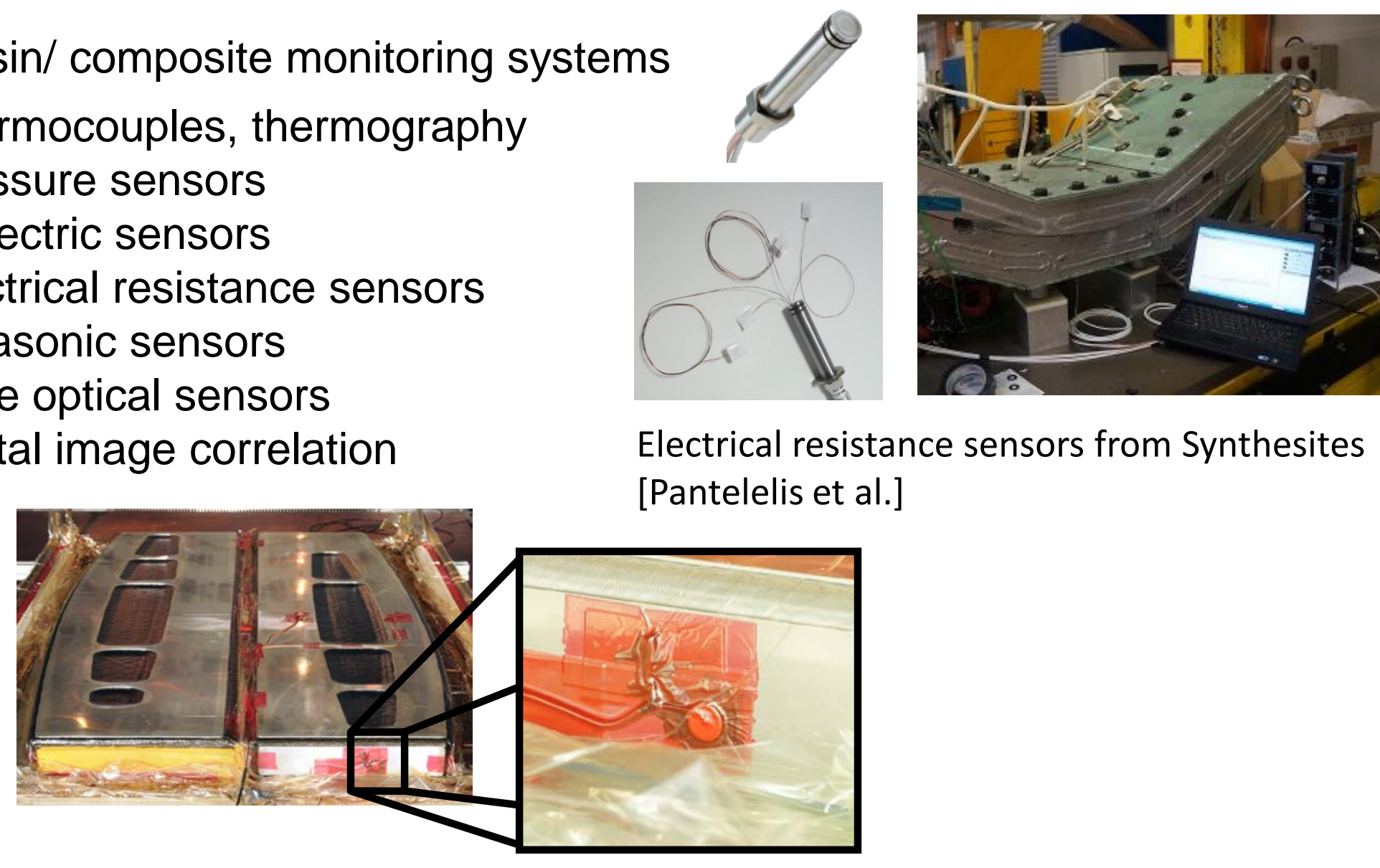

Ultrasonic sensor system from DLR [Liebers et al.] 


\section{Resin Infiltration and Curing Monitoring}

During measurement

- Feature storage within manufacturing database and digital life data sheet

- Direct feedback to online process simulation and optimization possible

Measures in case of deviations

- Adjust temperature profile

- Adjust pressure

- Open/ close valves

- Stop process

Impact

- In-situ NDI (filling, Tg, degree of cure, etc.)

- Reduction of process time and cost

- Increased understanding of materials and processes as well as efficient root cause analysis in case of deviations 


\section{Resin Infiltration and Curing Monitoring}

Challenges

- Effective sensor network (multiple sensors and multiple purpose)

- Sensor robustness under environmental conditions

- Accessibility within closed tools, autoclave

- Part damage by contacting or integrated systems

- Sensor and material model calibration considering uncertainties

- Real-time data and process analysis as well as forecast 


\section{Example:}

In-situ structural evaluation of of process induced distortions of a composite suspension blade (ECOMISE project)
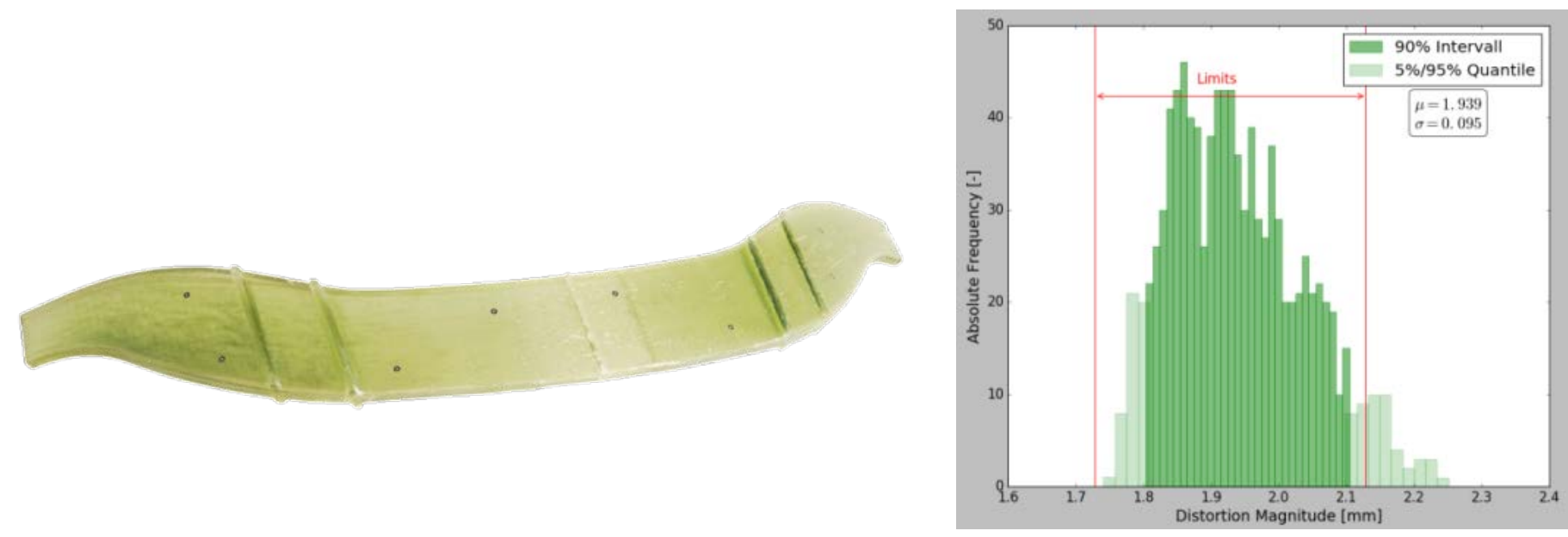


\section{In-situ evaluation of process induced distortions}

\section{Procedure}

1. Prior to Manufacturing

- Process and sensitivity analysis

- Surrogate modeling for in-situ process simulation and evaluation
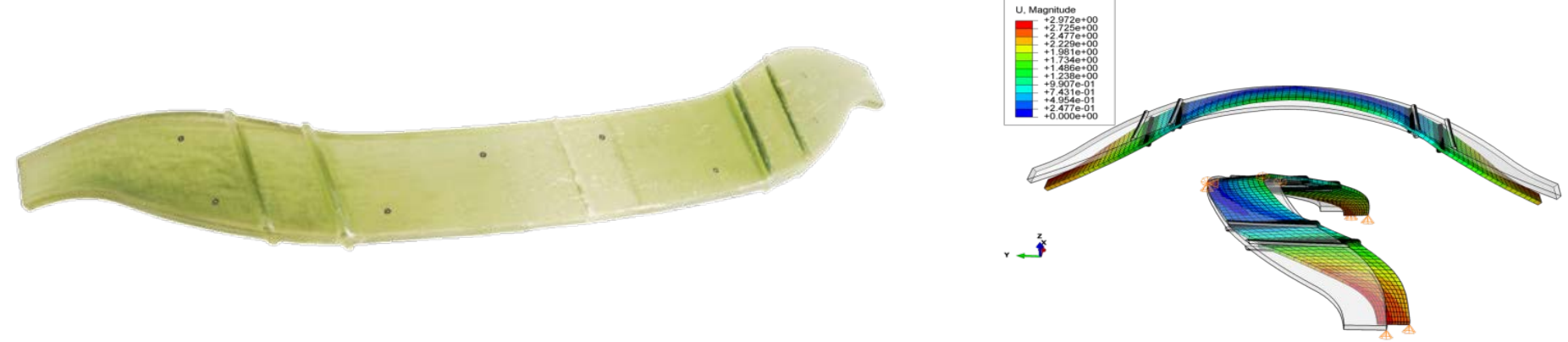

1. During Manufacturing

- Online process monitoring

- In-situ evaluation and process adjustment

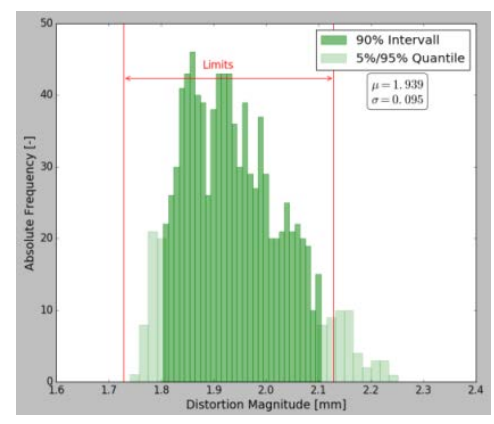




\section{In-situ evaluation of process induced distortions}

Industrial application (1. step, offline)

- Curing and distortion simulation
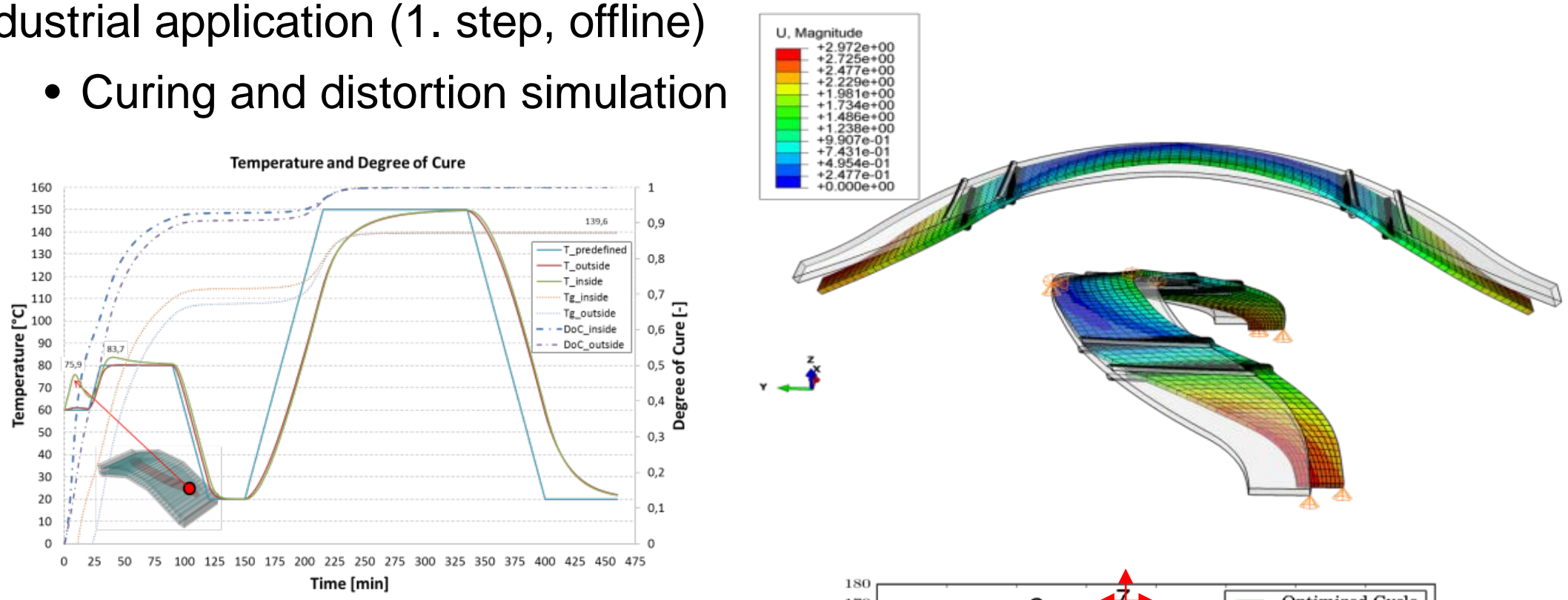

- MaxiMin-Design for varying material and process parameters,

- Evaluation of material \& process dependency

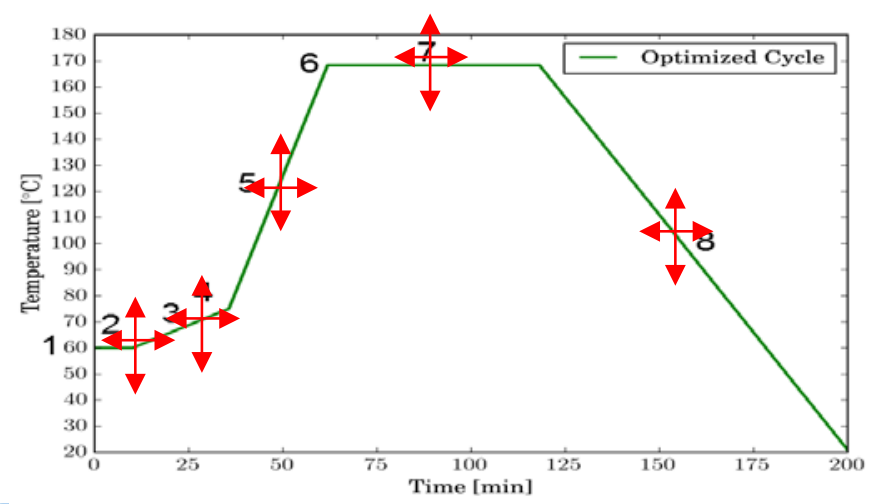




\section{In-situ evaluation of process induced distortions}

Industrial application (1. step, offline, cont.)

- Surrogate modeling of process induced distortion (Kriging and $\mathrm{RBF}$ ), capturing variations of material and process parameters, e.g.

- Coefficient of thermal expansion

- Tool temperature

- Injection time

- Time dependent cure cycle

- ...
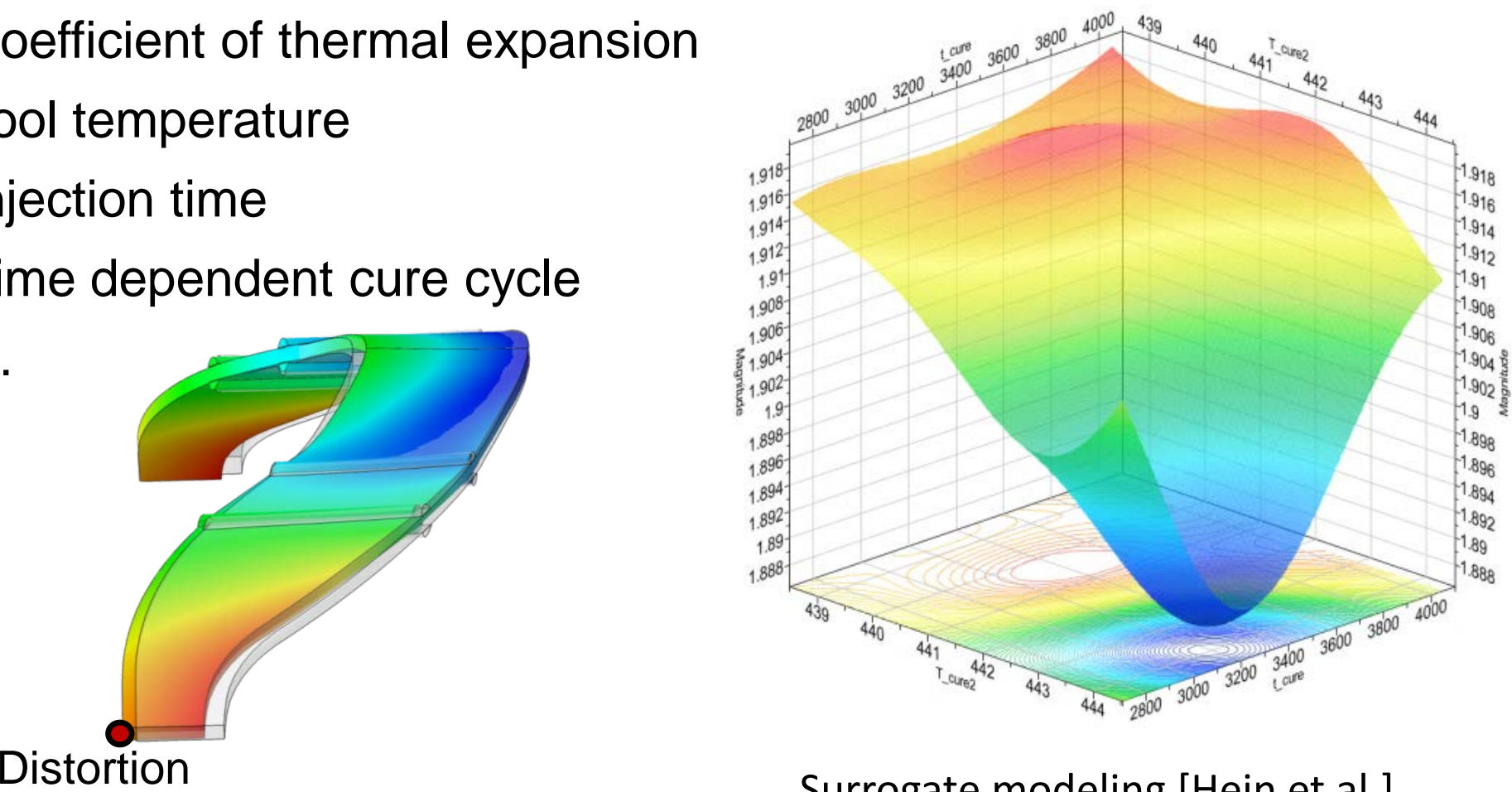

Surrogate modeling [Hein et al.] 


\section{In-situ evaluation of process induced distortions}

Industrial application (2. step, online)

- In-situ feedback of sensor data (temperature) and model update

- In-situ distortion or stress analysis and evaluation wrt. requirements

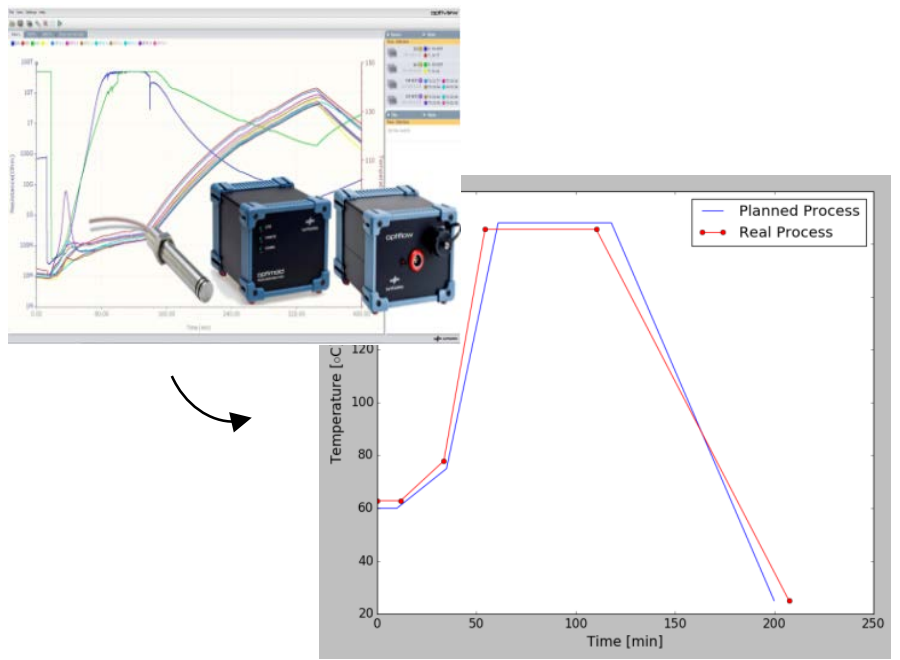

As-is cure cycle update with sensor data at time $t_{-} i$

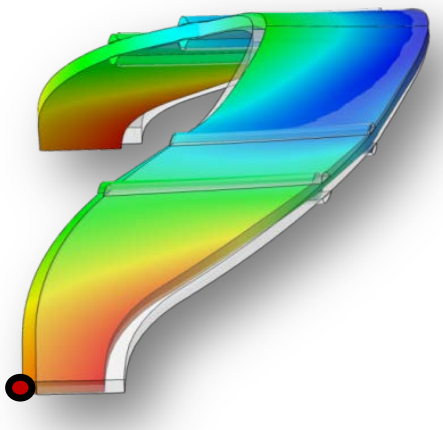

Prediction of distortion or stress at time t_final

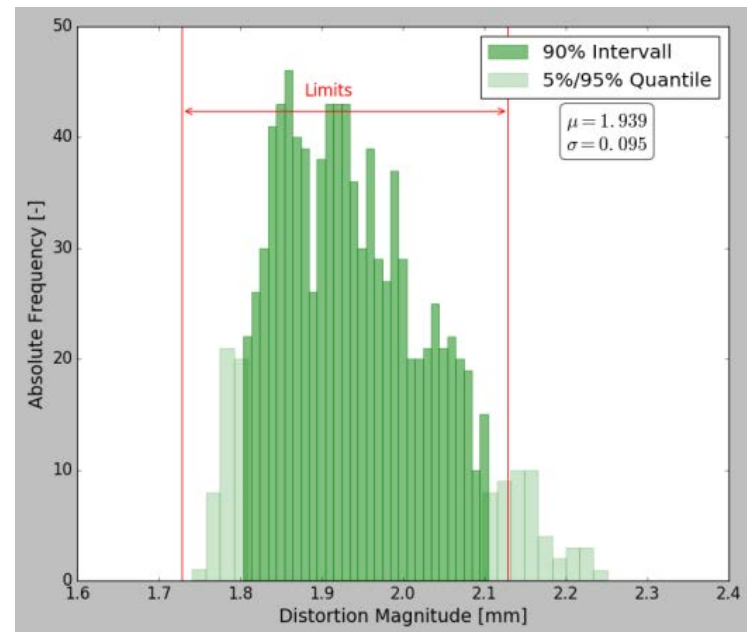

Tolerance check wrt. final process induced distortions

[Hein et al.] 


\section{Conclusion}

Variety of monitoring and evaluation systems available and under development (suited for different materials, manufacturing processes, parameters)

Increasing acceptance and application through industrial demonstrations

Enabling

- Increased phenomenological understanding, efficient root cause analysis

- Early defect detection and enhanced NDI

- Enhanced tolerance management and process control

- Semi-automated feedback to structural analysis for enhanced evaluation Impacts

- Fast ramp-up by less manufacturing trials

- Minimum cycle time and cost

- Reduced material and energy consumption as well as emissions

- Reduced concessions near to zero 


\section{Thank You}

The research leading to these results has received funding from European Community's FP7-2013-NMP-ICT-FoF (ECOMISE)

Contact

Dr.-Ing. Tobias Wille Head of Structural Mechanics Department Institute of Composite Structures and Adaptive Systems Phone: +49 531 295-3012

Mail: tobias.wille@dlr.de Web: www.DLR.de 